\title{
PHOSPHORESCENCE MICROWAVE PHOTOEXCITATION SPECTRUM OF 1,4-DIBROMONAPHTHALENE *
}

\author{
Paras N. PRASAD \\ Department of Chemistry, State University of New York at Buffalo, \\ Buffalo, New York 14214, USA
}

\author{
A.I. ATTIA \\ Dayton, Ohio 45433, USA \\ and

\begin{abstract}
A.H. FRANCIS
Department of Chemistry, University of Michigan. Ann Arbor, Michigan 48109, USA
\end{abstract}

Air Force Materials Laboratory, Wright-Patterson Air Force Base,

Received 1 September 1976

\begin{abstract}
The phosphorescence microwave photoexcitation spectrum of 1,4-dibromonaphthalene is obtained by monitoring the $\mathrm{X}$-trap phosphorescence at $20164 \mathrm{~cm}^{-1}$ and AM modulating the $2942 \mathrm{MHz}$ zero-field spin resonance. The comparative spectrum shows that the spin alignment of the emitting $T_{1}$ state is dependent upon the nature of the pumping state. The result is discussed in terms of the spin-orbit coupling routes and various intramolecular relaxation schemes.
\end{abstract}

\section{Introduction}

In the conventional triplet state phosphorescence microwave double resonance (PMDR) experiment [1], one modulates the zero field spin populations of the phosphorescent triplet via resonant microwave radiation and detects intensity changes in the features of the phosphorescence spectrum. Such studies have been found to be extremely helpful $[1,2]$ in assigning the spin-orbital symmetry of triplet states and determining the spin-orbit coupling routes from the nature of spin activity and the spin-alignment $\neq$ produced.

The technique of phosphorescence microwave photoexcitation has been used by Wilkerson et al. [3] to

* Supported in part by NSF Grant No. DMR-75-02628 and in part by Air Force Material Laboratory, Contract F3361573-C-5048.

* We are using "spin alignment" to represent a non-Boltzmann distribution of spin populations in spin substates created by a selective relaxation scheme. investigate the mechanism of host-guest energy transfer in chemically mixed crystals. This technique has application to the study of various intermolecular and intramolecular relaxation schemes which populate the lowest triplet state. In phosphorescence microwave photoexcitation spectroscopy (PMPS) [3] one monitors a single line of the phosphorescence spectrum while modulating the triplet zero field spin-state populations via resonant-amplitude or frequency modulated microwave radiation. Both the ac and dc components of the modulated phosphorescence intensity are recorded as a function of excitation wavelength. The ratio of the ac component to the dc component provides information about the relative spin alignment as a function of the excitation energy. This information is helpful in understanding the nature of the interactions leading to energy cascade from an absorbing state to the lowest emitting triplet state.

In this paper we present an application of this technique [3] in studying the nature of intramolecular re- 
laxation processes which populate the lowest triplet. The system investigated is 1,4-dibromonaphthalene in which the lowest triplet can be pumped by excitation into more than one higher state $[4,5]$. The result obtained indicates a sensitivity of the spin alignment to the overall excitation routes.

\section{Experimental}

Single crystal samples of 1,4-dibromonaphthalene, prepared by standard Bridgman methods from extensively zone refined reagent grade material, were mounted in a slow wave helix structure affixed to the end of a length of 0.141 stainless steel rigid cryogenic coaxial cable and emersed directly in superfluid helium at approximately $2 \mathrm{~K}$. The source of $20 \mathrm{~Hz}$ amplitude modulated microwave radiation was the output of a HewlettPackard 8690 Sweeper Mainframe and 8699B oscillator plug-in, amplified by a Servo 3003 microwave power amplifier. Microwave power levels of approximately $1 \mathrm{~W}$ were used in the experiments.

The continuum background source for the optical excitation spectrum was a $75 \mathrm{~W}$ PEK xenon lamp, monochromated with a McPherson 1/2-meter scanning monochromator set for a bandpass of $10 \AA$.

The modulated phosphorescence was dispersed in the first order of a 1 meter Jarrell-Ash recording monochromator equipped with an EMI 9558 QB refrigerated photomultiplier. The ac and dc components of the modulated fhosphorescence were separated by a conventional pissive $R C$ filter network and the dic component amplified by a Keithley 155 electrometer. The ac component was amplificd by a PAR 126 lock-in amplifier referenced in phase and frequency to the $20 \mathrm{~Hz}$ microwave modulation source. To obtain the ratio spectrum the output of the electrometer was divided by the output of the lock-in using an Intech A-733 single quadrant divider module. This device provides a maximum error of $0.5 \%$ in the dc/ac quotient, which is independent of the level of the input signals over a range of 0.1 to $10 \mathrm{~V} \mathrm{dc}$. Therefore, it is necessary to adjust the gains of the electrometer and lock-in amplifier to provide output levels within this range over the entire spectral range scanned. The experimental arrangement differs substantially from that of Wilkerson et al. [3] only with regard to the use of real-time normalization of the PMPS spectrum.

\section{Results}

Since ODMR signals coild not be observed when monitoring the pure exciton phosphorescence origin $\neq$, the present study was conducted by monitoring phosphorescence from the 1,4-dibromonaphthalene defect site (X-trap). The defect phosphorescence at 20164 $\mathrm{cm}^{-1}$ which is $28 \mathrm{~cm}^{-1}$ below the exciton origin was selected for study for the following reasons: (i) zero field parameters for this defect site are fairly close to that for the 1,4-dibromonaphthalene monomer in the isotopic mixed crystals $[7,8]$. (ii) The relative radiative rates of the spin-states of this defect emission are comparable to those for the exciton emission [8]. These observations indicate that this defect site is only a mildly perturbed site. Furthermore, as this defect site gives rise to a shallow trap for the triplet emission it acts mainly as a feeder to deeper traps by the mechanism of trap-to-trap migration. For this reason, spinlattice relaxation due to communication between defect sites of various trap depths is not expected to play an important role. This is because once the excitation leaves this defect site for the deeper traps, it does not have large probability at $2 \mathrm{~K}$ to return back to another defect site of the same energy.

The photoexcitation spectrum obtained by monitoring the defect phosphorescence at $20164 \mathrm{~cm}^{-1}$ is shown in fig. 1. The lowest triplet state $\left(T_{1}\right)$ is populated [5] by excitation into vibronic levels of $T_{1}, a$ higher triplet state $\left(\mathrm{T}_{2}\right)$ with origin at approximately $3356 \AA$, or by excitation into the lowest singlet state $\left(S_{1}\right)$ with origin at approximately $3293 \AA$. The phosphorescence microwave photoexcitation spectrum (PMPS) obtained by AM modulation at $20 \mathrm{~Hz}$ of the $2942 \mathrm{MHz}$ spin transition is also shown in fig. 1. The ratio of the two photoexcitation spectra which gives the relative spin alignment versus the excitation wavelength is shown in fig. 2 . It may be seen from fig. 2 that the spin alignment is sensitive to the nature of the pumping state. Specifically, excitation into $S_{1}$ creates no detectable spin alignment.

The oscillator strength of the $S_{1} \leftarrow S_{0}$ transition is several orders of magnitude greater than that of either the $T_{2} \leftarrow S_{0}$ or $T_{1} \leftarrow S_{0}$ transitions. Therefore, in single crystals of 1,4-dibromonaphthalene, singlet excitation

\footnotetext{
Spin transitions on the excitation transition have been observed in a magnetic field. See ref. [6].
} 


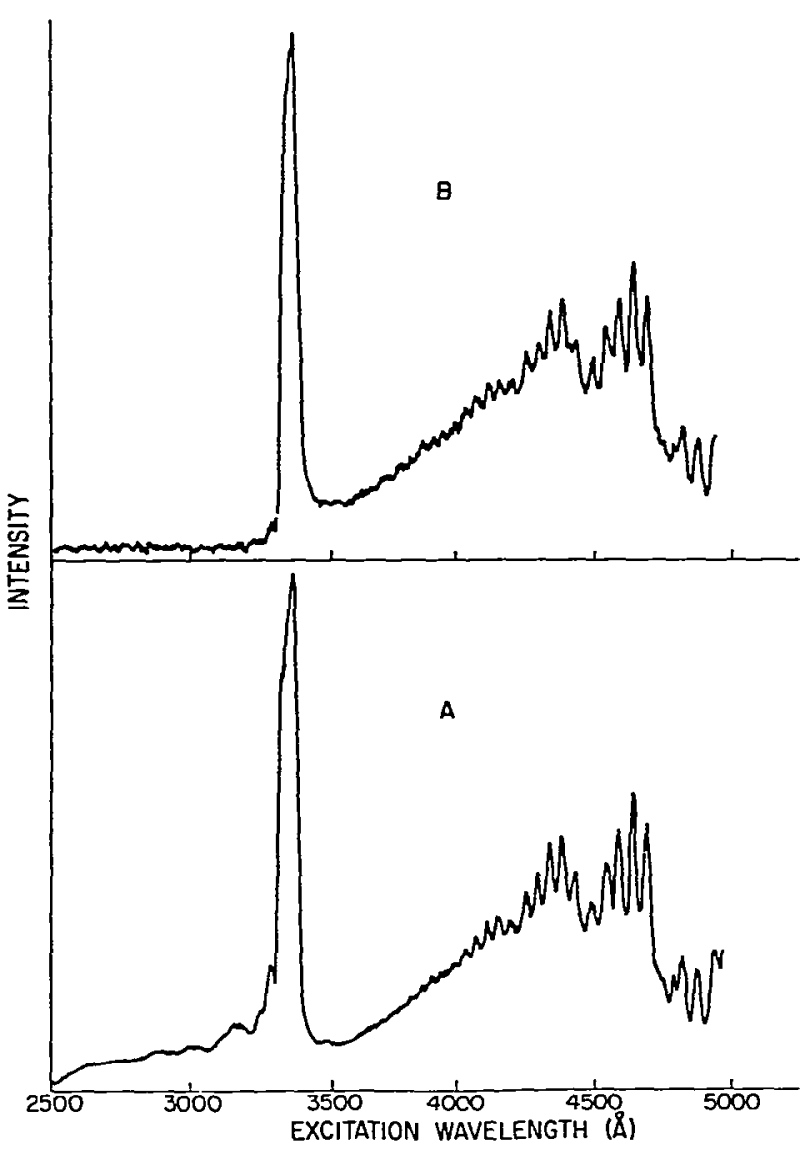

Fig. 1. $A$ is the normal photoexcitation spectrum and $B$ is the microwave photoexcitation spectrum (PMPS) both obtained by monitoring the $20164 \mathrm{~cm}^{-1}$ defect phosphorescence of 1,4-dibromonaphthalene neat crystal. These spectra were obtained at $1.8 \mathrm{~K}$ with the excitation bandpass of $10 \mathrm{~A}$.

is confined to a thin layer of molecules near the surface of the crystal, whereas triplet excitation occurs predominately in the bulk of the sample. For this rea- son, the PMPS spectrum of the pure crystal does not sample the same set of sites for singlet excitation as for triplet excitation. Therefore, the possibility was considered that the loss of spin alignment upon excitation into the first singlet state might be wholly or in part due to surface perturbations rather than to intramolecular effects. In order to investigate this possibility, the microwave modulated photoexcitation spectrum of 1,4-dibromonaphthalene in durene was recorded. The normal photoexcitation spectrum showed that the main pumping state for $T_{1}$ phosphorescence of 1,4-dibromonaphthalene was its own singlet, $S_{1}$ rather than the host (durene) excited states. In dilute (1\%) chemically mixed crystals we may reasonably anticipate that surface effects of the type described above will be negligible for the guest singlet absorption. The PMPS spectrum, on the other hand, again shows that in this chemically mixed crystal the excitation into the 1,4-dibromonaphthalene singlet does not produce any detectable spin alignment. The resonance was too weak to obtain a clear (above the noise level) PMPS spectrum in the durene host. We, thus, conclude that the loss of spin alignment in $T_{1}$ upon $S_{1}$ excitation is predominately determined by the dynamics of intramolecular intersystem crossing.

We have noted that $T_{2}$ excitation and direct $T_{1}$ (vibronic) excitation produce sinilar $T_{1}$ spin alignment with regard to both sign and magnitude of the observed signal. This observation suggests that $T_{1}$ and $T_{2}$ have the same active spin-state.

We also observe that in the same system that excitation into various vibronic levels of $T_{I}$ produces the same spin alignment. There is a strong vibronic origin at $256 \mathrm{~cm}^{-1}$ from the origin but no different behavior is observed for this.

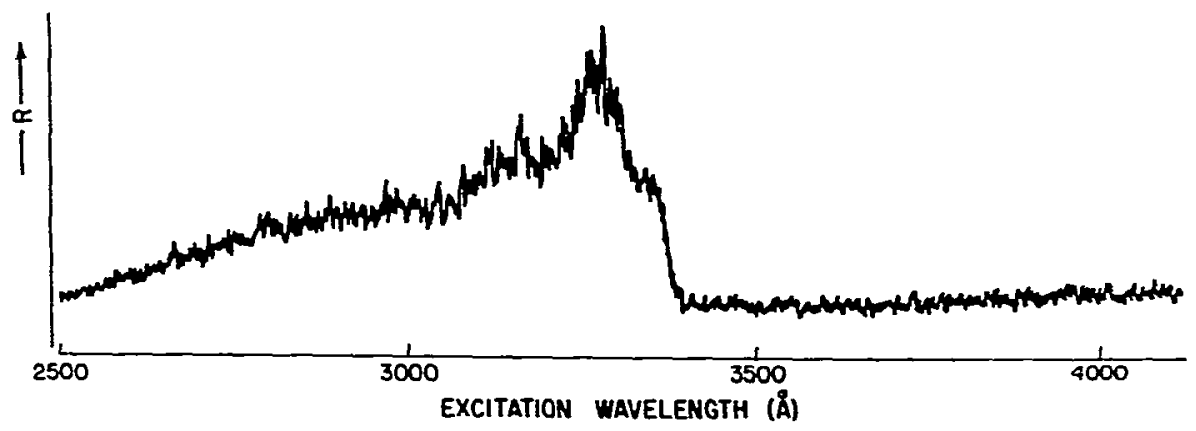

Fig. 2. The ratio $R$ is defined by: $R=$ dc photoexcitation component/ac photoexcitation component. The experimental value of $R$ as a function of the excitation wavelength has been obtained by dividing the two spectra electronically. 


\section{Discussion}

In a planar molecule like 1,4-dibromonaphthalene having a $\mathrm{C}_{2 \mathrm{v}}$ molecular symmetry, the excited $\pi \pi^{*}$ configurations correspond to $A_{1}$ and $B_{2}$ symmetries [9], if we choose the coordinate axes such that $X, Y$ and $Z$ correspond respectively to the out-of-plane, short and long molecular axes. $T_{1}$ state has been characterized [10] as ${ }^{3} B_{2}$ with predominant spin activity in $\tau_{z}$ spin state. The monitored $2942 \mathrm{MHz}$ spin transition corresponds to a $\tau_{z} \leftrightarrow \tau_{x}$ transition $[7,8]$. This makes the phosphorescence very sensitive to the 2942 $\mathrm{MHz}$ microwave resonance.

First we will analyze our results with triplet excitations. No attempt has been made in the past to make a symmetry assignment for the $T_{2}$ state. Our result shows that $T_{2}$ has the same spin activity as $T_{1}$. This suggests that if $T_{2}$ is a $\pi \pi^{*}$ state, it is also ${ }^{3} B_{2}$ in orbital symmetiy. In a ${ }^{3} A_{1}$ state $\tau_{z}$ is not spin-active. On the basis of a correlation [9] with the predicted orbitally allowed $\mathrm{T}_{2}$ transition of naphthalene one would expect the $T_{2}$ of 1,4-dibromonaphthalene to have $A_{1}$ orbital symmetry. However, there are nearby triplet states in naphthalene [9], which could give rise to a state of $B_{2}$ symmetry for 1,4-dibromonaphthalene. It may be that the halogen perturbation changes their relative radiative rates so that the observed transition is of $B_{2}$ symmetry. A definite explanation of this is not possible at this stage especially in view of the fact that the $T_{2}$ state of naphthalene has not been experimentally characterized.

The observation that the excitation into $256 \mathrm{~cm}^{-1}$ vibronic band of $T_{1}$ gives a spin alignment of similar magnitude and sign indicates that the $\tau_{z}$ spin state is also active in this vibronic origin (orbital symmetry $B_{1}$ ). This observation compares well sith the results of Castro and Hochstrasser [10] who have established that the vibronic origin of exciton transition is endowed with $\tau_{z}$ activity by spin-orbit vibronic coupling to the ${ }^{1} \mathrm{~B}_{2}\left(\pi \pi^{*}\right)$ state.

Finally we would like to discuss a nearly complete loss of spin alignment observed with $S_{1}$ excitation, although within the framework of the present experimental data it is not possible to provide a definite explanation for this observation. On the basis of a correlation with the first singlet of naphthalene one would suspect the symmetry of $S_{1}$ of 1,4-dibromonaphthalene to be $\mathbf{A}_{1}$. In such a case an intersystem crossing involving a direct spin-orbit coupling will selectively popu- late the $\tau_{x}$ spin state. However, if in addition to this direct coupling, a spin-orbit vibronic coupling using an intermediate $B_{1}$ state is equally important in the intersystem crossing, this will populate $\tau_{z}$ spin-state. The net result will be a loss of spin-alignment for the $\tau_{z} \leftrightarrow \tau_{z}$ resonance. This is in agreement with our experimental finding. In the alternative assignment ${ }^{1} \mathbf{B}_{2}$ for the lowest singlet state, no direct spin-orbit coupling exists between ${ }^{1} B_{2}$ and ${ }^{3} B_{2}$ states. However, the suggested $[10,11]$ strong spin-orbit vibronic coupling between ${ }^{1} B_{2}$ and ${ }^{3} B_{2}$ through the intermediate ${ }^{1} \mathbf{B}_{1}$ state should selectively populate the $\tau_{z}$ level of $T_{1}$ leading to a favorable spin-alignment for PMPS. Our result on the singlet excitation, thus, appears to favor the ${ }^{1} A_{1}$ assignment for $S_{1}$ with both the direct spinorbit as well as a spin-orbit vibronic coupling playing comparable roles in the intersystem crossing to $T_{1}$. We note that our finding on the intersystem crossing from $S_{1}$ is in qualitative agreement with that in ref. [8].

\section{Acknowledgement}

We wish to thank Professor R.M. Hochstrasser for eniightening discussions.

\section{References}

[1] M.A. El-Sayed, in: Excited states, Vol. 1, ed. E.C. Lim (Academic Press, New York, 1974) p. 35, and references therein.

[2] A.H. Francis and C.B. Harris, J. Chem. Phys. 57 (1970) 1050.

[3] A.K. Wilkerson, J.B. Chodak and M.A. El-Sayed, Chem. Phys. Letters 25 (1974) 464.

[4] G. Castro and R.M. Hochstrasser, J. Chem. Phys. 47 (1967) 2241.

[5] R.M. Hochstrasser and J.D. Whiteman, J. Chem. Phys. 56 (1972) 5945.

[6] R. Schmidberger and H.C. Wolf, Chem. Phys. Letters 16 (1972) 402.

[7] A.H. Zewail, Ph.D. Thesis, The University of Pennsylvania (1974).

[8] A.M. Nishimina, A.H. Zewail and C.B. Harris, J. Chem. Phys. 63 (1975) 1919.

[9] S.P. McGlymn, T. Azumi and M. Kinoshita, Molecular spectroscopy of the triplet state (Prentice-Hall, Englewood Cliffs, 1969).

[10] G. Castro and R.M. Hochstrasser, J. Chem. Phys. 48 (1968) 637.

[11] M.A. El-Sayed, J. Chem. Phys. 43 (1965) 2864. 